\title{
Role of abscisic acid in strigolactone- induced salt stress tolerance in arbuscular mycorrhizal Sesbania cannabina seedlings
}

\author{
Cheng-Gang Ren, Cun-Cui Kong and Zhi-Hong Xie
}

\begin{abstract}
Background: Strigolactones (SLS) are considered to be a novel class of phytohormone involved in plant defense responses. Currently, their relationships with other plant hormones, such as abscisic acid (ABA), during responses to salinity stress are largely unknown.

Results: In this study, the relationship between SL and ABA during the induction of $\mathrm{H}_{2} \mathrm{O}_{2}$ - mediated tolerance to salt stress were studied in arbuscular mycorrhizal (AM) Sesbania cannabina seedlings. The SL levels increased after ABA treatments and decreased when ABA biosynthesis was inhibited in AM plants. Additionally, the expression levels of SL-biosynthesis genes in AM plants increased following treatments with exogenous $\mathrm{ABA}$ and $\mathrm{H}_{2} \mathrm{O}_{2}$. Furthermore, $\mathrm{ABA}$-induced $\mathrm{SL}$ production was blocked by a pre-treatment with dimethylthiourea, which scavenges $\mathrm{H}_{2} \mathrm{O}_{2}$. In contrast, $\mathrm{ABA}$ production was unaffected by dimethylthiourea. Abscisic acid induced only partial and transient increases in the salt tolerance of TIS108 (a SL synthesis inhibitor) treated AM plants, whereas SL induced considerable and prolonged increases in salt tolerance after a pre-treatment with tungstate.
\end{abstract}

Conclusions: These results strongly suggest that $A B A$ is regulating the induction of salt tolerance by $S L$ in AM S. cannabina seedlings.

Keywords: Strigolactones, Abscisic acid arbuscular mycorrhizal, Sesbania cannabina, Photosynthesis, Salt stress

\section{Background}

Saline-alkali stress is a serious ecological problem that negatively impacts plant survival, development, and productivity [1]. To survive such stress, plants have established beneficial associations with several microorganisms present in the rhizosphere that can alleviate the stress symptoms [2]. One of the most intensively studied and widespread mutualistic plant-microorganism associations involves arbuscular mycorrhizal (AM) fungi. About $80 \%$ of terrestrial plants, including most leguminous species, are able to establish this type of symbiotic relationship with fungi of the division Glomeromycota [3]. Indeed, AM symbiosis increases resistance to soil salinity in diverse host plants, such as maize, tomato, and lettuce [4], although the underlying mechanisms are not well characterized [5]. Sesbania

\footnotetext{
* Correspondence: zhxie@yic.ac.cn

Key Laboratory of Biology and Utilization of Biological Resources of Coastal

Zone, Yantai Institute of Coastal Zone Research, Chinese Academy of

Sciences, Yantai 264003, China
}

cannabina, which is a soil-improving legume, is highly adaptable to different adverse climatic conditions, including salinity, drought, and waterlogging stresses. Therefore, developing S. cannabina-AM fungi symbiotic relationships might represent a good strategy for improving the fertility of saline soils to enable the growth of agriculturally important crops [6].

Establishing a functioning symbiotic relationship with an AM fungus requires a precise coordination between the partners based on a finely regulated molecular dialogue [7]. The molecular dialogue occurring during the so-called pre-symbiotic stage starts when the host plant produces and exudes strigolactones (SLs) into the rhizosphere. Strigolactones are recognized by AM fungi with an uncharacterized receptor that stimulates hyphal growth and branching, thereby increasing the probability of encountering the host roots [8]. In accordance with their role as signaling molecules in the rhizosphere, SLs are 
mainly produced in the roots and have been detected in the root extracts of monocotyledonous and dicotyledonous plants [9]. Strigolactones are biosynthetically derived from carotenoids [10] via a sequential oxidative cleavage by two carotenoid cleavage dioxygenases (CCD7 and CCD8), and are apocarotenoids [11], as is abscisic acid (ABA). Once synthesized, SLs are transported acropetally to the shoot or are exuded into the rhizosphere by the $A B C$ transporter PDR1, which was first identified in Petunia hybrida [12]. The F-box protein MAX2 and the $\alpha / \beta$ fold hydrolase D14/DAD2 are the main candidate components of the SL-perception complex in higher plants $[13,14]$. Additionally, MAX2 has diverse roles, including in plant responses to abiotic stress [15]. The importance of SLs during the initial stages of mycorrhizal colonizations is widely recognized. Moreover, emerging evidence indicates that SLs may also affect the subsequent steps of the symbiotic relationship developing in response to environmental stimuli, such as salt and drought stresses $[4,16]$. We recently observed that SLs can induce salt stress tolerance in AM $S$. cannabina seedlings that is regulated by the generation of $\mathrm{H}_{2} \mathrm{O}_{2}$ as a signaling molecule [17].

Abscisic acid is an important plant hormone with a critical role in the regulation of salt stress responses [18]. Exposure to stresses, such as salinity, induces the accumulation of $\mathrm{ABA}$, resulting in increased tolerance [19]. Several lines of evidence have revealed that ABA induces the accumulation of $\mathrm{H}_{2} \mathrm{O}_{2}$, which plays an important role in ABA signaling [20]. Moreover, SLs and $A B A$ are critical for the regulation of salt stress responses and the establishment of symbiotic relationships between host plants and AM fungi $[4,21]$. Recent studies unveiled the links between ABA and SLs in a number of physiological processes. Exogenous ABA may enhance the accumulation of SLs, especially under stress conditions [22]. Additional studies have demonstrated that SL metabolism and its effects on ABA levels are seemingly opposite in roots and shoots under stress [23]. However, it is unclear whether SL and ABA are involved in a synergistic effect during stress responses. These results suggest the possible relationship between SL and $\mathrm{ABA}$ during the induction of plant stress tolerance is complex.

The mechanisms by which SLs enhance plant stress tolerance have so far been largely uncharacterized. Our previous studies confirmed that $\mathrm{H}_{2} \mathrm{O}_{2}$ induces SL production and is critical for SL-induced salt stress tolerance in AM S. cannabina seedlings. In the present study, we inoculated S. cannabina seedlings with AM fungi and monitored the $\mathrm{H}_{2} \mathrm{O}_{2}$ level, $\mathrm{ABA}$ content, and $\mathrm{SL}$ accumulation to examine the role of $\mathrm{ABA}$ and the relationship between $\mathrm{ABA}$ and $\mathrm{H}_{2} \mathrm{O}_{2}$ in the $\mathrm{AM}$ fungus- induced increase in SL production in response to salt stress conditions.

\section{Results \\ Effects of ABA levels on strigolactone accumulation}

In $S$. cannabina seedlings inoculated with an AM fungus, the root colonization rate steadily increased as seedlings grew and differed significantly between sampling times (Table 1). Additionally, the ABA concentration was the highest in salt treated S. cannabina seedlings and it was higher in salttreated AM S. cannabina seedlings than in the water-treated controls (Fig. 1), indicating that salt stress may have triggered ABA biosynthesis both in AM and non-AM S. cannabina seedlings. Moreover, a bioassay of SL showed that the $P$. ramosa germination rate increased significantly in response to the root extract of salt-stressed AM S. cannabina seedlings [17], as did the endogenous SL levels $(\mathrm{m} / z 383, \mathrm{~m} / z 356$, and $m / z$ 317) (Additional file 1: Table S1).

To determine whether ABA affects SL generation, we examined the changes in SL levels in AM S. cannabina seedlings after $A B A$ and tungstate treatments. As shown in Fig. 4b, ABA did not accumulate in seedlings treated with tungstate, which prevents the formation of $A B A$ from $A B A$ aldehyde by inhibiting $A B A$-aldehyde oxidase [24]. First, the ABA treatment increased SL levels in AM seedlings but not in non-AM seedlings. Second, the germination bioassay revealed that SL accumulated in saltstressed AM S. cannabina seedlings 10 days after the ABA treatment. However, this ABA-induced increase was not observed in seedlings pre-treated with $5 \mu \mathrm{M}$ TIS108, a SL synthesis inhibitor (Fig. 2). These results suggested that ABA was important for the AM fungusinduced SL synthesis under salt stress conditions. In contrast, when S. cannabina seedlings were treated with an ABA synthesis inhibitor tungstate, the SL content decreased significantly, but the SL level could be recovered by an ABA co-treatment. It also showed that $\mathrm{H}_{2} \mathrm{O}_{2}$ could affect SL accumulation in AM S. cannabina seedlings (Fig. 2). Considered together, these results suggested that ABA induced the accumulation of SLs in AM S. cannabina seedlings.

\section{Abscisic acid affects the expression of strigolactone biosynthesis/signaling genes in S. cannabina seedlings} To analyze the molecular mechanisms underlying the induction of SL synthesis in AM S. cannabina seedlings

Table 1 Colonization of Sesbania cannabina seedling roots by Funneliformis mosseae. Values are presented as the mean \pm standard error of triplicate samples. Data were analyzed using Duncan's multiple range test at $p<0.05$. WAS: weeks after sowing

\begin{tabular}{llll}
\hline Time & 3 WAS & 5 WAS & 7 WAS \\
\hline AMF colonization & $8.6 \pm 0.54 \mathrm{c}$ & $26.3 \pm 3.1 \mathrm{~b}$ & $45.8 \pm 3.73 \mathrm{a}$ \\
\hline
\end{tabular}




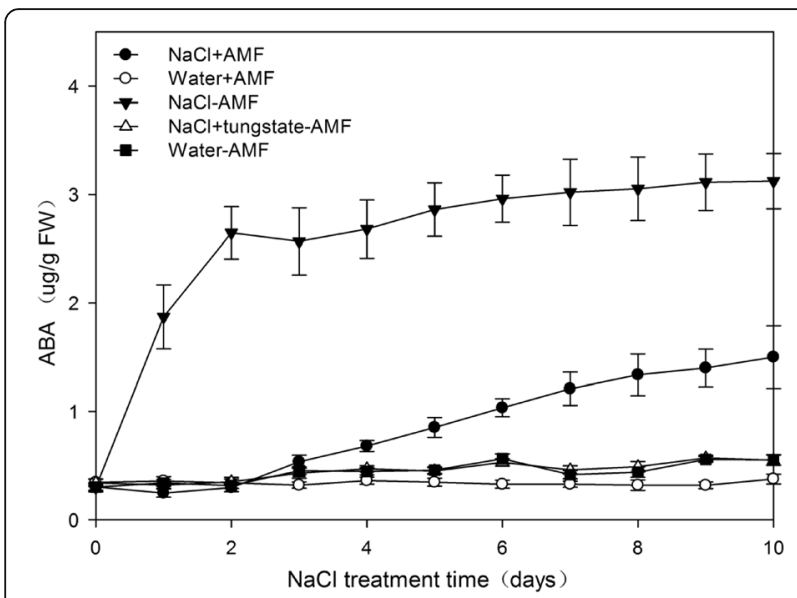

Fig. 1 Abscisic acid (ABA) concentration at 1-day intervals in Sesbania cannabina seedlings under different salt concentrations with and without an inoculation with an arbuscular mycorrhizal fungus. Values are presented as the mean of three independent experiments. The error bar represents the standard error

under salt stress, we examined the effects of $\mathrm{ABA}$ and $\mathrm{H}_{2} \mathrm{O}_{2}$ on the expression of CCD7, CCD8, and MAX2 homologs in $S$. cannabina seedlings. The expression levels of two SL-biosynthesis genes, $C C D 7$ and $C C D 8$, were up-regulated by $\mathrm{NaCl}$, exogenous $\mathrm{ABA}$, and $\mathrm{H}_{2} \mathrm{O}_{2}$

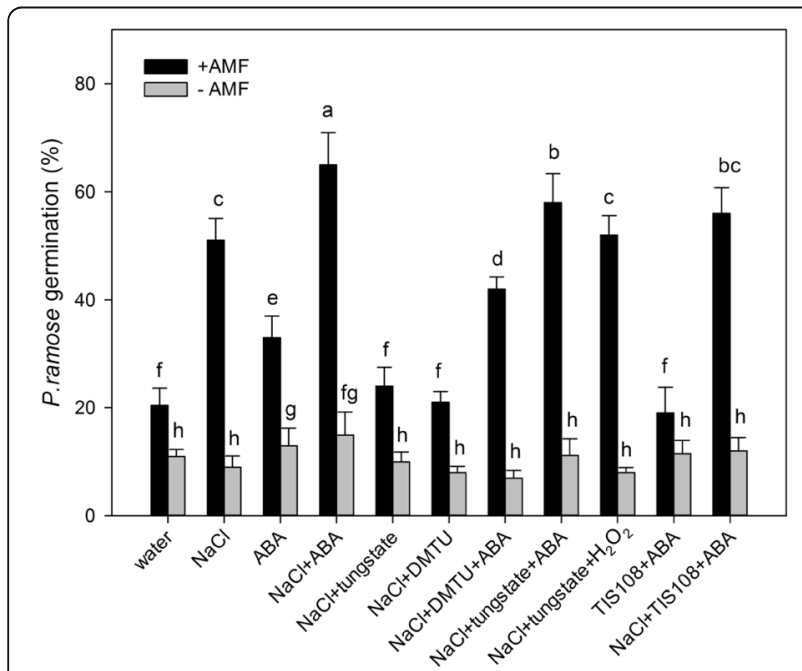

Fig. 2 Effect of hydrogen peroxide $\left(\mathrm{H}_{2} \mathrm{O}_{2}\right)$ inhibitors and abscisic acid $(\mathrm{ABA})$ inhibitors on strigolactone (SL) accumulation in arbuscular mycorrhizal Sesbania cannabina seedlings after a 10-day treatment. Germination of Phelipanche ramosa seeds induced by S. cannabina seedling root extracts. Treatments comprised inhibitors (5 mM DMTU, $5 \mathrm{mM}$ tungstate, and 2 MM TIS108) and $200 \mathrm{mM} \mathrm{NaCl}$, which were applied 1 day before the inoculation with the arbuscular mycorrhizal fungus. Values are presented as the mean of three independent experiments. The error bar represents the standard error. Data were analyzed using Duncan's multiple range test. Different letters above the error bars indicate statistical significance at $p<0.05$
(Fig. 3). Furthermore, tungstate and DMTU, an $\mathrm{H}_{2} \mathrm{O}_{2}$ scavenger, suppressed the expression of $C C D 7$ and $C C D 8$ in salt-stressed AM S. cannabina roots. Additionally, seedlings pre-treated with TIS108, which blocks SL biosynthesis, exhibited up-regulated expression of $C C D 7$ and CCD8. We also observed that salt stress, exogenous ABA, and $\mathrm{H}_{2} \mathrm{O}_{2}$ enhanced the expression of the SL-signaling gene, $M A X 2$, in AM S. cannabina shoots. It is worthy to note that $C C D 7$ and $C C D 8$ mainly expressed in root tissue, while $M A X 2$ in shoot tissue.

\section{Interactions between $\mathrm{ABA}$ and $\mathrm{H}_{2} \mathrm{O}_{2}$ related to strigolactone-induced salt tolerance}

An earlier study concluded that elevated $\mathrm{H}_{2} \mathrm{O}_{2}$ levels are involved in SL-induced salt stress tolerance [17]. Furthermore, several studies have revealed that ABA can induce the increased production of $\mathrm{H}_{2} \mathrm{O}_{2}$ (Kwak et al. [20]). Therefore, determining whether ABA helps to increase $\mathrm{H}_{2} \mathrm{O}_{2}$ production in AM S. cannabina seedlings is warranted. To test this possibility, we pre-treated $S$. cannabina seedlings with tungstate, which prevented the accumulation of ABA and considerably decreased $\mathrm{H}_{2} \mathrm{O}_{2}$ levels in AM S. cannabina seedlings (Fig. 4). Additionally, ABA-induced SL production was inhibited by the pretreatment with DMTU (Fig. 2). These results implied that an increase in ABA content is required for $\mathrm{H}_{2} \mathrm{O}_{2}$ to induce $\mathrm{SL}$ production. In contrast, when we pre-treated seedlings with DMTU, we observed no significant effect on the increase in $\mathrm{ABA}$ content under salt stress conditions (Fig. 4b). Thus, ABA appears to function upstream of $\mathrm{H}_{2} \mathrm{O}_{2}$ in AM S. cannabina seedlings.

\section{Dependency of ABA biosynthesis for strigolactone- induced salt tolerance}

To study the relationship between ABA- and SL-induced salt tolerances, we analyzed the effects of TIS108, a specific inhibitor of SL biosynthesis, and tungstate, an ABA biosynthesis inhibitor, on SL- and ABA-induced salt tolerances in AM S. cannabina seedlings. All plants not treated with salt had similar ФPSII values (approximately 0.57 ), while those exposed to $200 \mathrm{mM} \mathrm{NaCl}$ had significantly decreased ФPSII values at 2 and 8 days, especially in seedlings treated with TIS108 and tungstate (Fig. 5a, c). These results suggested that a defect in either ABA or SL accumulation decreased the salt stress tolerance of the seedlings. A pretreatment with ABA or GR24 (synthetic SL analog) at 2 or 8 days prior to the exposure to salt stress significantly increased ФPSII values in both control and AM plants. Moreover, GR24 restored the salt tolerance of tungstate-treated plants, while $\mathrm{ABA}$ restored the salt tolerance of TIS108-treated plants at 2 days after its application, but was ineffective at 8 days after its application (Fig. 5). Thus, SL was able to rescue the salt stress tolerance of ABA-deficient plants, while 

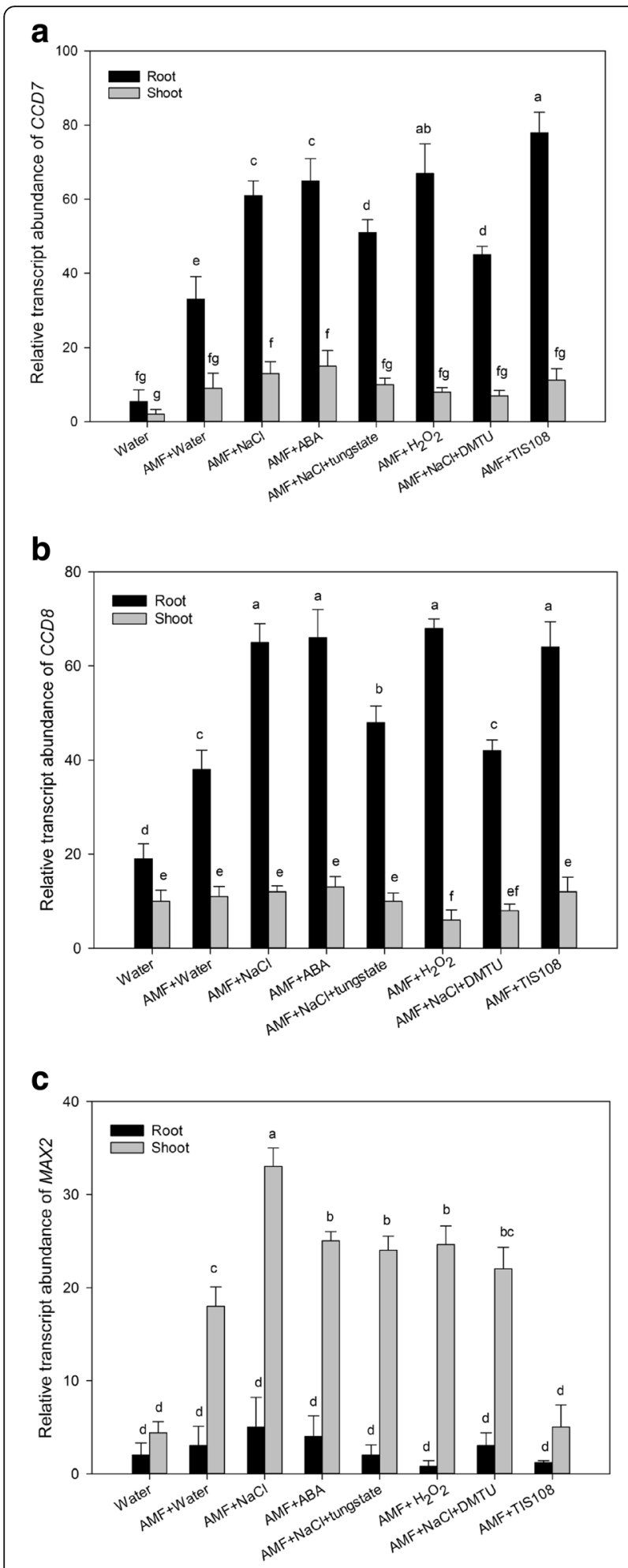

Fig. 3 Relative expression levels of SL-biosynthesis/signaling genes in Sesbania cannabina seedlings after a 10-day treatment. a Relative expression levels of CCD7. $\mathbf{b}$ Relative expression levels of CCD8. c Relative expression levels of MAX2. The expression level of each gene was normalized against that of the $S$. cannabina tubulin gene [41] and is provided relative to the control expression level $(=1)$. Data are presented as the mean \pm standard deviation of three biological replicates. Data were analyzed using Duncan's multiple range test. Different letters above the error bars indicate statistical significance at $p<0.05$

ABA could only partially and transiently rescue the defective stress tolerance of the tungstate-treated plants. This observation implied that SL might function downstream of $\mathrm{ABA}$ in plant stress responses.

Furthermore, ABA-induced salt tolerance was effectively blocked by a pre-treatment with DMTU (Fig. 6), while SL-induced salt tolerance was regulated by DMTU [17]. These results suggested that $\mathrm{H}_{2} \mathrm{O}_{2}$ was also responsible for the observed ABA- and SL-induced salt tolerances.

\section{Discussion}

Plants are sessile in nature. Therefore, to counter biotic and abiotic stresses, such as pathogens, drought, waterlogging, extreme temperatures, salinity, and many others, plants have evolved intricate signaling mechanisms composed of multiple components including plant hormones. Research conducted in the last decade have added SLs to the growing list of plant hormones involved in responses to environmental stresses [25]. In the present study, we revealed that SLs can be regulated by ABA production in S. cannabina seedlings (Figs 1,2). Given the common metabolic precursor shared by ABA and SL, a potential crosstalk between these two hormones was recently proposed, although these hormones appear to be negatively correlated. For example, SL levels reportedly decreased, while ABA content increased in tomato and lettuce plants grown under drought conditions in the absence of AM symbiosis [16]. We also observed that $A B A$ levels increased rapidly under salt stress conditions in plants not colonized by an AM fungus, whereas SL production was very low (Fig. 1). Therefore, this negative correlation might represent a general plant strategy to cope with water-related stresses in the absence of mycorrhization. Conversely, a positive correlation between ABA and SLs has been observed in stress-treated mycorrhizal plants $[4,16]$. Similarly, increasing ABA and SL levels were detected in $S$. cannabina seedlings under salt stress conditions in the presence of the AM fungus (Fig. 1). Additionally, ABA enhanced $P$. ramosa germination, whereas tungstate had the opposite effect (Fig. 2). The different relationships between SL and ABA in AM and non-AM S. cannabina respectively may indicate that the $\mathrm{ABA}$ catabolism was 


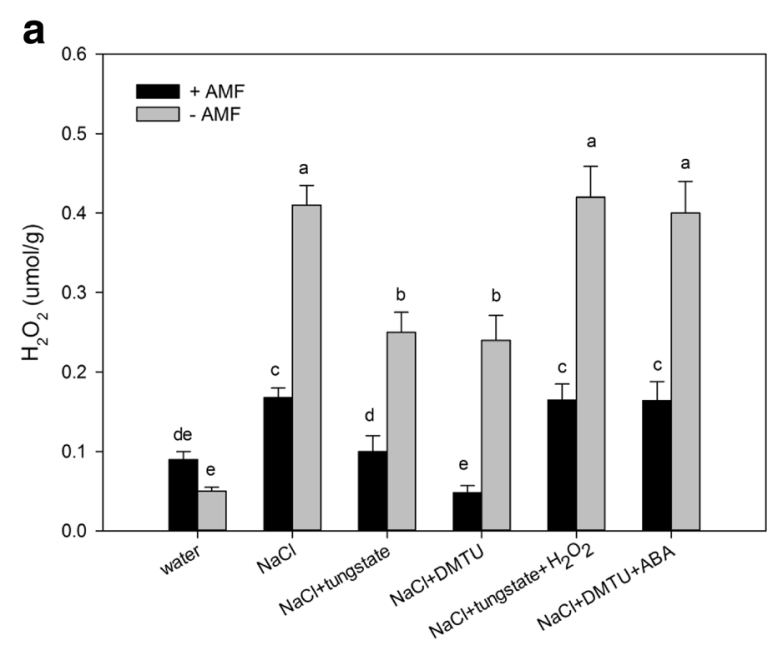

b

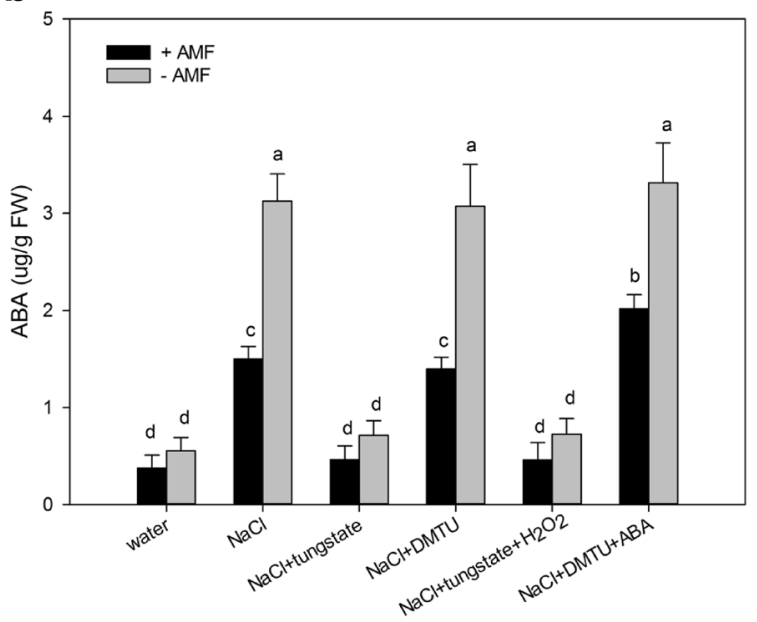

Fig. 4 Hydrogen peroxide $\left(\mathrm{H}_{2} \mathrm{O}_{2}\right)$ concentration and abscisic acid (ABA) accumulation in Sesbania cannabina seedlings after 10-day treatments. a $\mathrm{H}_{2} \mathrm{O}_{2}$ content. b Abscisic acid level. Treatments comprised inhibitors (5 $\mathrm{mM}$ DMTU and $5 \mathrm{mM}$ tungstate) and exogenous signaling molecules ( $10 \mathrm{mM} \mathrm{H}_{2} \mathrm{O}_{2}$ and $100 \mu \mathrm{M} \mathrm{ABA}$ ). Values are presented as the mean of three independent experiments. The error bar represents the standard error. Data were analyzed using Duncan's multiple range test. Different letters above the error bars indicate statistical significance at $p<0.05$

intensively altered by AM inoculation. Overall, it seem that the increase in ABA concentration induced by the AM fungus contributed to the increased accumulation of SLs in salt-stressed S. cannabina seedlings.

Our previous results suggested that $\mathrm{H}_{2} \mathrm{O}_{2}$-induced SL production is due to induced/activated NADPH oxidase and is important for SL-induced salt stress tolerance. In this study, we confirmed that ABA has a key role in the production of $\mathrm{H}_{2} \mathrm{O}_{2}$, which regulates the accumulation of SLs. We revealed that $\mathrm{H}_{2} \mathrm{O}_{2}$ production was substantially decreased by a pre-treatment with tungstate (Fig. 4a). Moreover, ABA accumulation was unaffected by a pre-treatment with DMTU (Fig. 4b). Previous investigations also concluded that ABA causes $\mathrm{H}_{2} \mathrm{O}_{2}$ to accumulate in the apoplast, which is dependent on NADPH oxidase and is important for ABA signaling $[20,26]$. These results suggest that ABA may act upstream of $\mathrm{H}_{2} \mathrm{O}_{2}$ in the signaling pathway of AM $S$. cannabina seedlings. Furthermore, we provided evidence that $\mathrm{H}_{2} \mathrm{O}_{2}$ is important for ABA-induced SL production. We also observed that SL production was considerably decreased by a pre-treatment with tungstate or DMTU (Fig. 2). An ABA treatment partly restored the $P$. ramosa germination rate in DMTU-treated mycorrhizal plantlets. Simultaneously, an $\mathrm{H}_{2} \mathrm{O}_{2}$ treatment significantly restored the $P$. ramosa germination rate of tungstate-treated mycorrhizal plantlets (Fig. 2). Taken together, these results implied that ABA induced SL accumulation in AM $S$. cannabina seedlings, which may be partially dependent on increased $\mathrm{H}_{2} \mathrm{O}_{2}$ levels.

In this study, we confirmed that ABA is involved in the SL-induced salt tolerance of S. cannabina seedlings inoculated with an AM fungus. Exposure to tungstate decreased SL levels and ФPSII values in salt-stressed AM S. cannabina seedlings (Figs 2, 5a, c). Furthermore, an ABA treatment significantly restored the $P$. ramosa germination rate in TIS108-treated mycorrhizal plantlets, indicating that the ABA-induced salt stress tolerance of AM S. cannabina seedlings required the accumulation of SL (Fig. 2). The relationship between SL and ABA during plant responses to abiotic stresses has been rarely studied. Many investigations have considered ABA as the 'abiotic stress hormone' because its biosynthesis is rapidly induced by exposures to environmental stresses, especially water-related stresses such as drought and salinity [27-29]. The reported increase in the ABA content of AM plants subjected to abiotic stress likely contributes to the enhanced tolerance of these plants to environmental stresses [4, 30]. Additionally, increased SL levels were detected in lettuce plants grown in the presence of the AM fungus Rhizophagus irregularis under salt stress conditions [4]. A similar trend has been observed in drought-treated lettuce and tomato plants [16]. These observations combined with our results indicate that SL-induced salt stress tolerance of AM plants may be mediated by a complex set of signal transduction pathways with $\mathrm{ABA}$ as a common signaling molecule.

\section{Conclusions}

In summary, we uncovered a dynamic interplay between SLs, ABA, and $\mathrm{H}_{2} \mathrm{O}_{2}$ produced in response to an AM fungus that contributed to the salt stress tolerance of $S$. cannabina seedlings. Following the perception of the ABA signal, $\mathrm{H}_{2} \mathrm{O}_{2}$ was rapidly produced, which then increased the accumulation of SLs, and ultimately enhanced the salt 

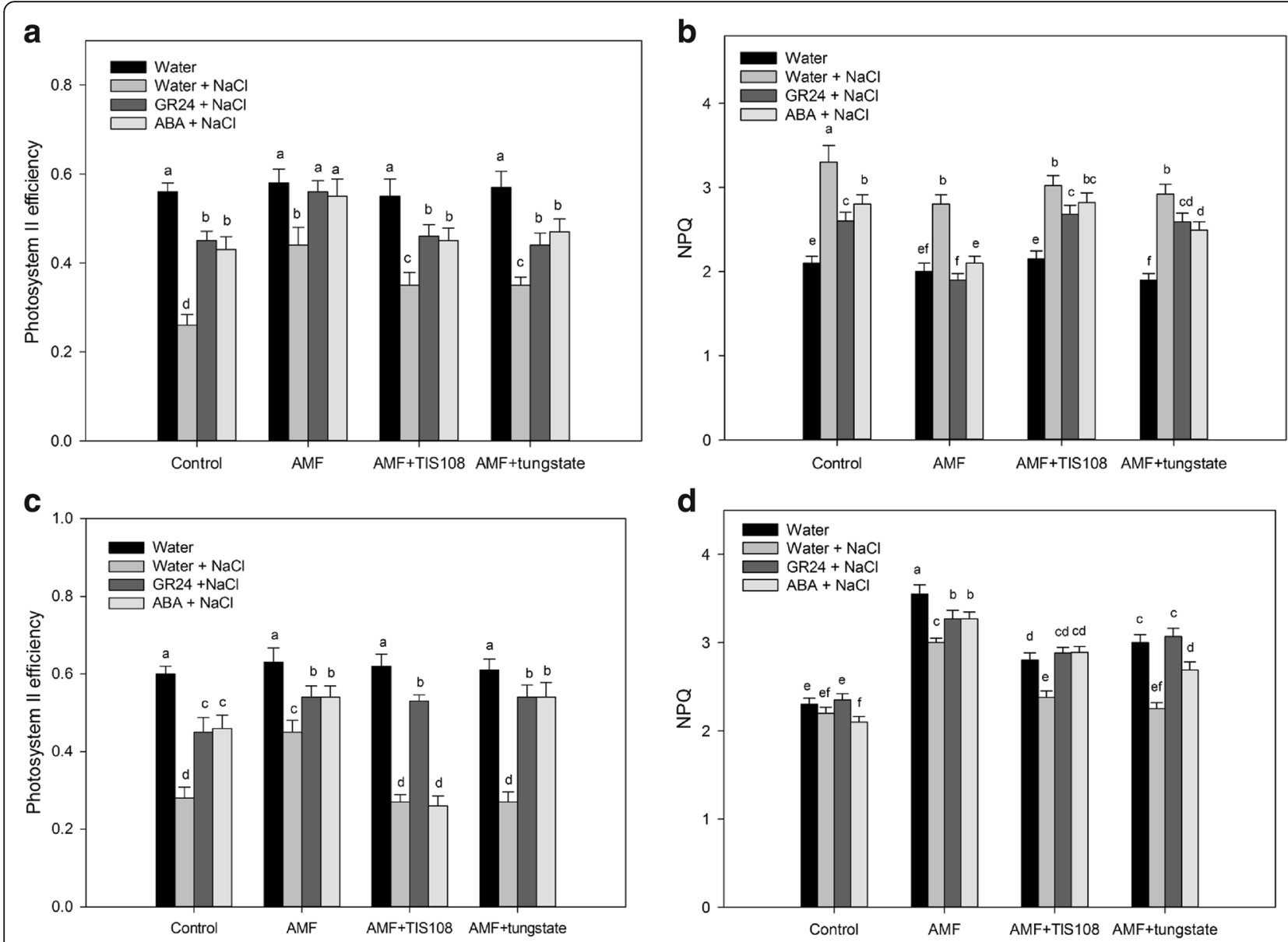

Fig. 5 Effects of strigolactone (SL) and abscisic acid (ABA) levels on photosynthetic parameters in salt-stressed Sesbania cannabina seedlings. a Photosystem II efficiency (DPSII) values of S. cannabina seedlings after 2 days under salt stress conditions. $\mathbf{b}$ Non-photochemical quenching of chlorophyll fluorescence (NPQ) of S. cannabina seedlings after 2 days under salt stress conditions. c Photosystem II efficiency values of S. cannabina seedlings after 8 days under salt stress conditions. $\mathbf{d}$ Non-photochemical quenching of chlorophyll fluorescence of $S$. cannabina seedlings after 8 days under salt stress conditions. Exogenous signaling molecules ( $1 \mu \mathrm{M}$ GR24 and $100 \mu \mathrm{M}$ ABA) were applied 1 day before the $S$. cannabina seedlings were exposed to salt stress conditions. Inhibitors ( $5 \mathrm{mM}$ tungstate and $2 \mu \mathrm{M}$ TIS108) were applied 1 day before the exogenous signaling molecules were applied. Values are presented as the mean of six independent experiments. The error bar represents the standard error. Data were analyzed using Duncan's multiple range test. Different letters above the error bars indicate statistical significance at $p<0.05$

stress tolerance of the seedlings. Additional studies are needed to provide the genetic evidence of the involvement of $\mathrm{ABA}$ in $\mathrm{H}_{2} \mathrm{O}_{2}$-induced SL generation and to identify the critical signaling components between SL production and salt stress responses in S. cannabina seedlings inoculated with an AM fungus. Such studies will help to elucidate the molecular mechanism underlying the ABA- and SL-induced salt tolerance of AM plants.

\section{Methods}

\section{Plant materials and treatments}

Sesbania cannabina (Retz.) Pers. seeds were obtained from the Shandong Academy of Agricultural Sciences, Shandong, China. Before sowing, the seeds were surface- sterilized in 5\% sodium hypochlorite for $5 \mathrm{~min}$ and rinsed several times with distilled water. The seeds were germinated at $28^{\circ} \mathrm{C}$ in distilled water and then sown in trays containing autoclaved zonolite. After 2 weeks, individual seedlings were transferred to $1-\mathrm{L}$ pots containing autoclaved zonolite inoculated with $10 \mathrm{~g}$ inoculum (approximately 121 spores). The original inoculum [AM fungus Funneliformis mosseae] was propagated in potcultured Trifolium repens plants for 8 weeks, and included infected roots, hyphae, spores, and substrates. The growth conditions were as follows: $12 \mathrm{~h}$ photoperiod, $25 / 17{ }^{\circ} \mathrm{C}$ (day/night), and light intensity of $600 \mu \mathrm{mol} \mathrm{m} \mathrm{m}^{-2} \mathrm{~s}^{-1}$. Three-week-old seedlings were used for all treatments with $200 \mathrm{mM} \mathrm{NaCl}$ solutions. The reagents used as 


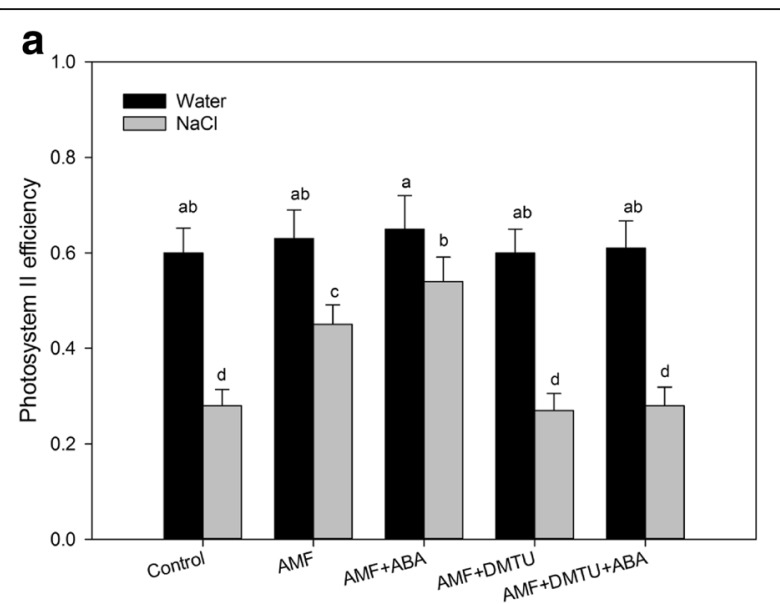

b

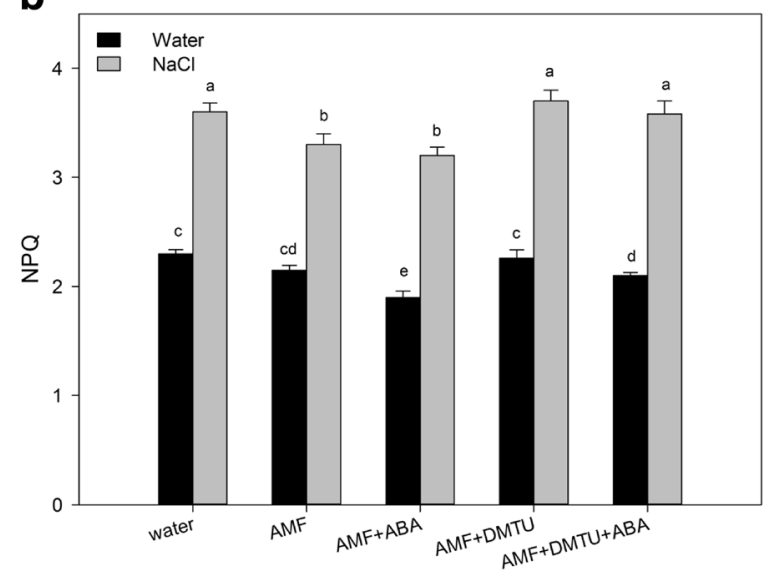

Fig. 6 Effect of abscisic acid (ABA) and $\mathrm{H}_{2} \mathrm{O}_{2}$ inhibitors on the photosynthetic parameters of arbuscular mycorrhizal Sesbania cannabina seedlings under salt stress conditions. a Photosystem II efficiency (DPSII) values of S. cannabina seedlings after 8 days under salt stress conditions. b Non-photochemical quenching of chlorophyll fluorescence (NPQ) of S. cannabina seedlings after 8 days under salt stress conditions. Exogenous signaling molecules and inhibitors comprised $100 \mu \mathrm{M}$ ABA and 5 mM DMTU. Values are presented as the mean of six independent experiments. The error bar represents the standard error. Data were analyzed using Duncan's multiple range test. Different letters above the error bars indicate statistical significance at $p<0.05$

specific scavengers or inhibitors $\left[5 \mathrm{mM}\right.$ DMTU $\left(\mathrm{H}_{2} \mathrm{O}_{2}\right.$ scavenger) [31], $5 \mathrm{mM}$ sodium tungstate [32], and $2 \mu \mathrm{M}$ TIS108 (the most potent and specific SL biosynthesis inhibitor) [33]] were purchased from Sigma-Aldrich (St Louis, MO, USA). Additionally, $10 \mathrm{mM} \mathrm{H}_{2} \mathrm{O}_{2}, 100 \mu \mathrm{M}$ ABA, and $1 \mu \mathrm{M}$ GR24 (synthetic SL analog) were used as exogenous signaling molecules [34]. Plantlet leaves were sprayed with $100 \mu \mathrm{L}$ exogenous signaling molecule or inhibitor solution. Treatments were completely randomized and replicated three times. An equal volume of distilled water was applied as the control treatment. Unless stated otherwise, inhibitors were applied $24 \mathrm{~h}$ before the exogenous signaling molecules were applied.

\section{Photosynthetic parameters}

Gas exchange and modulated chlorophyll fluorescence parameters were simultaneously detected using a LI6400XTR Portable Photosynthesis System (Li-Cor, Lincoln, NE, USA) equipped with a 6400-40 Leaf Chamber Fluorometer (Li-Cor). The leaves were incubated in darkness for $20 \mathrm{~min}$ before being analyzed. The minimal fluorescence level of the dark-adapted leaves was measured using a

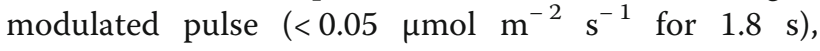
while the maximal fluorescence level was measured after applying a saturating actinic light pulse of $8000 \mu \mathrm{mol} \mathrm{m} \mathrm{m}^{-2} \mathrm{~s}^{-1}$ for $0.7 \mathrm{~s}$. The actinic light intensity was increased to $1000 \mu \mathrm{mol} \mathrm{m} \mathrm{m}^{-2} \mathrm{~s}^{-1}$ and then maintained for about $30 \mathrm{~min}$. The steady-state fluorescence yield was also recorded. A saturating actinic light pulse of $8000 \mu \mathrm{mol} \mathrm{m}{ }^{-2} \mathrm{~s}^{-1}$ for $0.7 \mathrm{~s}$ was then applied to induce the maximum fluorescence yield by temporarily inhibiting photosystem II (PSII) photochemical activities. The minimum steady-state fluorescence yield was determined during a brief interruption of the actinic light irradiation in the presence of far-red light $(\lambda=740 \mathrm{~nm})$. Finally, PSII efficiency (ФPSII) and non-photochemical quenching of chlorophyll fluorescence were calculated according to the method described by Maxwell and Johnson [35].

\section{Measurement of arbuscular mycorrhizal fungal colonization}

The percentage of roots colonized by mycorrhizal fungi was calculated using the gridline intersection method [36] after samples were stained with trypan blue [37].

\section{Measurement of $\mathrm{H}_{2} \mathrm{O}_{2}$}

Seedlings were harvested 10 days after treatments to measure the $\mathrm{H}_{2} \mathrm{O}_{2}$ content. The $\mathrm{H}_{2} \mathrm{O}_{2}$ concentration was determined by monitoring the absorbance of titanium peroxide at $415 \mathrm{~nm}$ following the method of Brennan and Frenkel [38]. One unit of $\mathrm{H}_{2} \mathrm{O}_{2}$ was defined as the chemiluminescence produced by the internal standard of $1 \mu \mathrm{M}$ $\mathrm{H}_{2} \mathrm{O}_{2} \mathrm{~g}^{-1}$ fresh weight.

\section{Extraction of ABA and HPLC analysis}

Seedlings collected for the ABA analysis were immediately frozen with liquid $\mathrm{N}_{2}$ and stored at $-80{ }^{\circ} \mathrm{C}$. Root tissue $(0.5 \mathrm{~g})$ was ground in a mortar and homogenized in $5 \mathrm{ml}$ pre-cooled $80 \%$ aqueous acetone $(4: 1, v / \mathrm{v})$ supplemented with $10 \mathrm{mg} / \mathrm{l}$ butylated hydroxytoluene. Extracts were eluted through a Sep Pak C18-cartridge (PerkinElmer, Waltham, MA, USA) to remove polar compounds and then purified using ethyl acetate and $\mathrm{NaHCO}_{3}$ as described by Sweetser and Vatvars [39]. After centrifuging samples at $14,000 \times \mathrm{g}$ for $30 \mathrm{~min}$ at $4{ }^{\circ} \mathrm{C}$, the supernatant was acidified to $\mathrm{pH} 3.0$ with $\mathrm{H}_{2} \mathrm{SO}_{4}$ and then treated three times with an equal volume of ethyl acetate. The extract phase was evaporated at $40{ }^{\circ} \mathrm{C}$ 
Table 2 Sequences of qRT-PCR primers used to analyze strigolactone-related genes

\begin{tabular}{lll}
\hline Gene & GenBank accession no. & Primers \\
\hline ScCCD7 & MF683952 & $5^{\prime}$-TGCTCCATCCACAACAGA-3' 5'-CTCCATAAGGCCAACACC-3' \\
SCCCD8 & MF683953 & $5^{\prime}$-TGGTCTAGCCACAAAGAA-3' 5'-ATGAACATGGGAAAGGAT-3' \\
SCMAX2 & MF683954 & $5^{\prime}$-TTGGATTAGGGTGGTGA-3' 5'-CTGAAGGTGCCGTGAGTA-3'
\end{tabular}

The nucleotide sequence reported in this table has been submitted to GenBank with accession numbers MF683952, MF683953 and MF683954

using the RE-100 rotary evaporator (Bibby Sterlin Ltd. , Stone Staffordshire, UK). The dried samples were re-dissolved in chromatography-grade acetonitrile with $0.1 \mathrm{M}$ acetic acid and then analyzed by HPLC using a Hypersil-ODS C18 column $(4.6 \mathrm{~mm} \times 250 \mathrm{~mm}, 5 \mu \mathrm{m})$. The mobile phase comprised acetonitrile: $0.03 \mathrm{M}$ acetic acid $(30: 70, \mathrm{v} / \mathrm{v})$ at a flow rate of $1 \mathrm{ml} / \mathrm{min}$. Abscisic acid was detected spectrophotometrically at $254 \mathrm{~nm}$ at $25^{\circ} \mathrm{C}$. The retention time was $13.37 \mathrm{~min}$. Abscisic acid standard curves $(20-3000 \mathrm{ng} / \mathrm{ml})$ were used to quantify ABA contents $\left(R^{2}=0.991\right)$.

\section{Extraction of strigolactones and quantification by a germination bioassay}

Root tissue $(0.5 \mathrm{~g})$ was collected and ground in a mortar with liquid nitrogen and then treated with $1 \mathrm{ml}$ ethyl acetate in a 3-ml glass tube. The tubes were vortexed and sonicated for $10 \mathrm{~min}$ in a Branson 3510 ultrasonic bath (Branson Ultrasonics, Danbury, CT, USA). Samples were then centrifuged at $4000 \times \mathrm{g}$ for $5 \mathrm{~min}$ in an MSE Mistral 2000 centrifuge (Mistral Instruments, Leicester, UK). The organic phase was carefully transferred to 1$\mathrm{ml}$ glass vials and stored at $-20{ }^{\circ} \mathrm{C}$ until used in the germination bioassays.

Strigolactones were identified by liquid chromatographytandem mass spectrometry using a Quattro LC mass spectrometer (Micromass, Manchester, UK) equipped with an electrospray source as previously described (Kong et al. [17]).

The germination bioassays with Phelipanche ramosa seeds were conducted according to the method developed by Yoneyama [40]. Approximately 20 surface-sterilized $P$. ramosa seeds were placed on 6- $\mathrm{mm}$ glass fiber discs (Whatman) and about 90 discs were incubated in a Petri dish $(9 \mathrm{~cm}$ diameter) lined with filter paper moistened with $6 \mathrm{ml}$ sterile Milli-Q water. The seeds required a 12-day pre-conditioning incubation at $21{ }^{\circ} \mathrm{C}$ in darkness before they became responsive to the germination stimulants. A sterile Petri dish ( $5 \mathrm{~cm}$ diameter) was lined with filter paper moistened with $50 \mu \mathrm{l}$ S. cannabina root extract, which was allowed to evaporate before the pre-conditioned seeds were added. The seeds were then treated with $650 \mu \mathrm{l}$ sterile Milli-Q water. The synthetic germination stimulant GR24 $\left(10^{-6} \mathrm{M}\right)$ and demineralized water were included as positive and negative controls in each bioassay. The Petri dishes were sealed, enclosed in polyethylene bags, and incubated at $25{ }^{\circ} \mathrm{C}$ in darkness for 7 days. The germinated and non-germinated seeds were counted using a stereoscope. Seeds were considered germinated if the radicle protruded through the seed coat.

\section{Quantitative real-time PCR}

Root samples from three seedlings were pooled to represent a single biological replicate. Total RNA was extracted from the pooled root samples using TRIzol (Invitrogen, Carlsbad, CA, USA) following the manufacturer's instructions. Three biological replicates were prepared. The extracted RNA was used as the template for reverse transcription reactions completed with the GoScript $^{\text {Tm }}$ Reverse Transcription System (Promega, Madison, WI, USA). Gene-specific primers were designed using the Primer Premier program (version 5.0) (Premier Biosoft International). The quantitative real-time (qRT)-PCR analysis was conducted with an iCycler iQ Real-time PCR Detection System (Bio-Rad) with three technical replicates. Each reaction was completed in a total volume of $20 \mu \mathrm{l}$, which included $0.2 \mu \mathrm{M}$ primer pairs, $2 \mu \mathrm{l}$ diluted cDNA, and $10 \mu \mathrm{l} 2 \times$ SYBR Green PCR Master Mix (TaKaRa Bio Inc., Dalian, China). The PCR program was as follows: $95^{\circ} \mathrm{C}$ for $30 \mathrm{~s} ; 40$ cycles of $95^{\circ} \mathrm{C}$ for $5 \mathrm{~s}, 58^{\circ}$ $\mathrm{C}$ for $15 \mathrm{~s}$, and $72{ }^{\circ} \mathrm{C}$ for $20 \mathrm{~s}$. Tubulin genes were used as the internal controls and relative gene expression levels were calculated using the $2^{-\Delta \Delta \mathrm{Ct}}$ method [41]. The sequences of the qRT-PCR primers are listed in Table 2.

\section{Statistical analysis}

All data were analyzed using Microsoft Excel (Redmond, WA, USA) and the values for each treatment are presented as the mean \pm standard deviation of three replicates. A one-way analysis of variance was completed with the SPSS Statistics 17.0 software (SPSS, Inc., Chicago, IL, USA). Duncan's multiple range test was used to compare mean values at the $p<0.05$ significance level.

\section{Additional file}

Additional file 1: Table S1 Effect of different treatments on the relative abundances of three SLs candidates m/z 383, m/z 356 and m/z 317 in S. cannabina seedlings extract.Data were analyzed using Duncan's multiple range test. Different letters indicate statistical significance at $p<0.05$. (XLSX 13 kb) 


\section{Abbreviations}

ABA: Abscisic acid; AM: Arbuscular mycorrhizal; CCD: Carotenoid cleavage dioxygenases; DMTU: Dimethylthiourea; $\mathrm{m} / \mathrm{z}$ : Mass to charge ratio; MAX2: More axillary growth2; NPQ: Non-photochemical quenching of chlorophyll fluorescence; SL: Strigolactone; TIS108: 6-phenoxy-1-phenyl-2-(1H1,2,4-triazol-1-yl) hexan-1-one; ФPSII: Photosystem II efficiency

\section{Acknowledgements}

We thank Professor Yong-Qing Ma (Northwest Agriculture and Forestry University) for supplying the Phelipanche ramosa seeds.

\section{Funding}

This work was financed by the National Natural Science Foundation of China (31601238 and 31570063), the High-tech Industrialization Cooperation Funds of Jilin province and the Chinese Academy of Science (2017SYHZ0007), Shandong Key Research and Development Program (2016CYJS05A01-1, 2017GSF17129), Shandong Key Scientific and Technological Innovation Program (2017CXGC0303), Mechanisms of marginal land productivity expansion and technologies of storing grain in land (KFZD-SW-112), Yantai Key Project of Research and Development Plan (2016ZH074).

\section{Availability of data and materials}

All data generated or analysed during this study are included in this published article [and its Additional file].

\section{Authors' contributions}

RCG designed and performed the experiment, analysed the data and wrote the manuscript. KCC perform extraction and identification the phytohormone using GC-MS. RCG and KCC participated in revisions of the manuscript. All authors have read the manuscript, given comments and ap proved the final version of the manuscript.

\section{Ethics approval and consent to participate}

Not applicable

\section{Competing interests}

The authors declare that they have no competing interests.

\section{Publisher's Note}

Springer Nature remains neutral with regard to jurisdictional claims in published maps and institutional affiliations.

\section{Received: 23 October 2017 Accepted: 24 April 2018} Published online: 03 May 2018

\section{References}

1. Manivannan P, Jaleel CA, Sankar B, Somasundaram R, Mural PV, Sridharan R, et al. Salt stress mitigation by calicium chloride in Vigna radiata (L.) Wilczek. Acta Biol Cracov Ser Bot. 2007:49:105-9.

2. Badri DV, Weir TL, Vander LD, Vivanco JM. Rhizosphere chemical dialogues: plant-microbe interactions. Curr Opin Biotechnol. 2009;20:642-50.

3. Smith SE, Read DJ. Mycorrhizal symbiosis. London: Academic Press; 2008

4. Aroca R, Ruiz-Lozano JM, Zamarreño AM, Paz JA, García-Mina JM, Pozo MJ, et al. Arbuscular mycorrhizal symbiosis influences strigolactone production under salinity and alleviates salt stress in lettuce plants. J Plant Physiol. 2013;170:47-55.

5. Ruiz-Lozano JM, Porcel R, Azcon R, Aroca R. Regulation by arbuscular mycorrhizae of the integrated physiological response to salinity in plants. New challenges in physiological and molecular studies. J Exp Bot. 2012:63:4033-44.

6. Ren CG, Bai YJ, Kong CC, Bian B, Xie ZH. Synergistic interactions between salt-tolerant rhizobia and arbuscular mycorrhizal fungi on salinity tolerance of Sesbania cannabina plant. J Plant Growth Regul. 2016; https://doi.org/10 1007/s00344- 016-9607-0.

7. Andreo-Jiménez B, Ruyter-Spira C, Bouwmeester H, López-Ráez JA Ecological relevance of strigolactones in nutrient uptake and other abiotic stresses, and in plant-microbe interactions below-ground. Plant Soil. 2015:394:1-19

8. Akiyama K, Matsuzaki K, Hayashi H. Plant sesquiterpenes induce hyphal branching in arbuscular mycorrhizal fungi. Nature. 2005;435:824-7.

9. Xie XN, Yoneyama K, Yoneyama K. The strigolactone story. Annu Rev Phytopathol. 2010;48:93-117.
10. López-Ráez JA, Charnikhova T, Gómez-Roldán V, Matusova R, Kohlen W, De $\operatorname{Vos} \mathrm{R}$, et al. Tomato strigolactones are derived from carotenoids and their biosynthesis is promoted by phosphate starvation. New. Phytologia. 2008;178:863-74.

11. Walter MH, Strack D. Carotenoids and their cleavage products:biosynthesis and functions. Nat Prod Rep. 2011;28:663-92.

12. Kretzschmar T, Kohlen W, Sasse J, Borghi L, Schlegel M, Bachelier JB, et al. A petunia $A B C$ protein controls strigolactone-dependent symbiotic signalling and branching. Nature. 2012:483:341-4.

13. Stirnberg P, Furner IJ, Leyser HMO. MAX2 participates in an SCF complex which acts locally at the node to suppress shoot branching. Plant J. 2007:50:80-94.

14. Zhao LH, Zhou XE, Wu ZS, Yi W, Xu Y, Li S, et al. Crystal structures of two phytohormone signal-transducing alpha/beta hydrolases: karrikin-signaling KAI2 and strigolactone-signaling DWARF14. Cell Res. 2013:23:436-9.

15. Bu QY, LV TX, Shen H, Luong P, Wang J, Wang Z, et al. Regulation of drought tolerance by the F-box protein MAX2 in Arabidopsis. Plant Physiol. 2014;164:424-39.

16. Ruiz-Lozano JM, Aroca R, Zamarreño ÁM, Molina S, Andreo-Jiménez B, Porcel $\mathrm{R}$, et al. Arbuscular mycorrhizal symbiosis induces strigolactone biosynthesis under drought and improves drought tolerance in lettuce and tomato. Plant Cell Environ. 2016;39:441-52.

17. Kong CC, Ren CG, Li RZ, Xie ZH, Wang JP. Hydrogen peroxide and strigolactones signaling are involved in alleviation of salt stress induced by arbuscular mycorrhizal fungus in sesbania cannabina seedlings. J Plant Growth Regul. 2017; https://doi.org/10.1007/s00344-017-9675-9

18. Ha CV, Leyva-González MA, Osakabe Y, Tran UT, Nishiyama R, Watanabe $Y$, et al. Positive regulatory role of strigolactone in plant responses to drought and salt stress. PNAS. 2014;111(14):851-6.

19. Zhu JK. Salt and drought stress signal transduction in plants. Annu Rev Plant Biol. 2002;53:247-73.

20. Kwak JM, Mori IC, Pei ZM, Leonhardt N, Torres MA, Dangl JL. NADPH oxidase AtrbohD and AtrbohF genes function in ROS-dependent ABA signaling in Arabidopsis. EMBO J. 2003;22:2623-33.

21. Pozo MJ, López-Ráez JA, Azcón C, García-Garrido JM. Phytohormones as integrators of environmental signals in the regulation of mycorrhizal symbioses. New Phytol. 2015;205:1431-6.

22. López-Ráez JA, Kohlen W, Charnikhova T, Mulder P, Undas AK, Sergeant MJ, et al. Does abscisic acid affect strigolactone biosynthesis? New Phytol. 2010;187:343-54.

23. Liu J, He H, Vitali M, Visentin I, Charnikhova T, Haider I, et al. Osmotic stress represses strigolactone biosynthesis in Lotus japonicus roots: exploring the interaction between strigolactones and ABA under abiotic stress. Planta. 2015;241:1435-51.

24. Hansen H, Grossmann K. Auxin-induced ethylene triggers abscisic acid biosynthesis and growth inhibition. Plant Physiol. 2000;124:1437-48.

25. Pandey A, Sharma M, Pandey GK. Emerging roles of Strigolactones in plant responses to stress and development. Front Plant Sci. 2016;7:434. https:// doi.org/10.3389/fpls.2016.00434

26. Pei Z, Murata Y, Benning G, Thomine S, Klusener B, Allen G, et al. Calcium channels activated by hydrogen peroxide mediate abscisic acid signaling in guard cells. Nature. 2000;406:731-4.

27. Hong $\mathrm{JH}$, Seah SW, Xu J. The root of ABA action in environmental stress response. Plant Cell Rep. 2013;32:971-83.

28. Osakabe Y, Osakabe K, Shinozaki K, Tran LSP. Response of plants to water stress. Front Plant Sci. 2014;5:86.

29. Peleg Z, Blumwald E. Hormone balance and abiotic stress tolerance in crop plants. Curr Opin Plant Biol. 2011;14:290-5.

30. Calvo-Polanco M, Sanchez-Romera B, Aroca R. Arbuscular mycorrhizal fungi and the tolerance of plants to drought and salinity. Symbiotic Endophytes. 2013:37:271-88

31. Xia XJ, Wang YJ, Zhou YH, Tao Y, Mao WH, Shi K, et al. Reactive oxygen species are involved in brassinosteroid-induced stress tolerance in cucumber. Plant Physiol. 2009;150:801-14.

32. Jiang MY, Zhang JH. Water stress-induced abscisic acid accumulation triggers the increased generation of reactive oxygen species and upregulates the activities of antioxidant enzymes in maize leaves. J Exp Bot. 2002:379:2401-10

33. Ito S, Umehara M, Hanada A, Kitahata N, Hayase $H$, Yamaguchi S, et al. Effects of triazole derivatives on strigolactone levels and growth retardation in rice. PLoS One. 2011; https://doi.org/10.1371/journal.pone0021723. 
34. Cuyper CD, Fromentin J, Yocgo RE, Keyser AD, Guillotin B, Kunert K, et al. From lateral root density to nodule number, the strigolactone analogue GR24 shapes the root architecture of Medicago truncatula. J Exp Bot. 2014;66:137-46.

35. Maxwell K, Johnson GN. Chlorophyll fluorescence: a practical quide. J Exp Bot. 2000;51:659-68.

36. Giovannetti M, Mosse B. An evaluation of techniques for measuring vesiculararbuscular mycorrhizal infection in roots. New Phytol. 1980;84:489-500.

37. Phillips JM, Hayman DS. Improved procedures for clearing roots and staining parasitic and vesicular-arbuscular mycorrhizal fungi for rapid assessment of infection. Trans Brit Mycol Soc. 1970;55:158-60.

38. Brennan $\mathrm{T}$, Frenkel $\mathrm{C}$. Involvement of hydrogen peroxide in the regulation of senescence in pear. Plant Physiol. 1977;59:411-6.

39. Sweetser PB, Vatvars A. High-performance liquid chromatographic analysis of abscisic acid in plant extracts. Anal Biochem. 1976;71:68-78.

40. Yoneyama K, Xie X, Sekimoto H, Takeuchi Y, Ogasawara S, Akiyama K, et al. Strigolactones, host recognition signals for root parasitic plants and arbuscular mycorrhizal fungi, from Fabaceae plants. New Phytol. 2008;179:484-94.

41. Ren CG, Kong CC, Yan K, Zhang H, Luo YM, Xie ZH. Elucidation of the molecular responses to waterlogging in Sesbania cannabina roots by transcriptome profiling. Sci Rep. 2017; https://doi.org/10.1038/s41598-017-07740-5.

Ready to submit your research? Choose BMC and benefit from:

- fast, convenient online submission

- thorough peer review by experienced researchers in your field

- rapid publication on acceptance

- support for research data, including large and complex data types

- gold Open Access which fosters wider collaboration and increased citations

- maximum visibility for your research: over $100 \mathrm{M}$ website views per year

At BMC, research is always in progress.

Learn more biomedcentral.com/submissions 